\title{
COVID-19: Pediatric Oral Health during and after the Pandemics
}

\author{
Valeria Luzzi ${ }^{1, *}$, Gaetano Ierardo ${ }^{2}$, Maurizio Bossù ${ }^{3}$ and Antonella Polimeni ${ }^{4}$ \\ 1 Department of Oral and Maxillo-Facial Science, "Sapienza" University of Rome, Rome, Italy; \\ valeria.luzzi@uniroma1.it \\ 2 Department of Oral and Maxillo-Facial Science, "Sapienza" University of Rome, Rome, Italy; \\ gaetano.ierardo@uniroma1.it \\ 3 Department of Oral and Maxillo-Facial Science, "Sapienza" University of Rome, Rome, Italy; \\ maurizio.bossu@uniroma1.it \\ 4 Department of Oral and Maxillo-Facial Science, "Sapienza" University of Rome, Rome, Italy; \\ antonella.polimeni@uniroma1.it \\ * Correspondence: valeria.luzzi@uniroma1.it; Tel.: +39-349-513-8825 (V.L.)
}

Received: date; Accepted: date; Published: date

\begin{abstract}
During the period of health emergency linked to the current COVID-19 pandemic, the management of children's oral health presents specific problems related to the infectious spread of the disease. These problems must be faced on the one hand by acting on the oral health prevention methods, and on the other by implementing specific protocols relating both to the conditions of oral pathologies that normally do not represent an emergency, and to those clinical situations that fall into the category of pediatric dental emergencies. In this perspective, in addition to defining rigorous and highly effective infection control protocols in the dental settings, it is of fundamental importance to work on remote communication and education aimed at maintaining the oral health of the children. This article, after an analysis of the risk factors from COVID-19 associated with pediatric dental treatment, presents a series of considerations on potential oral prevention strategies and on the management of emergency and non-emergency dental procedures in a context of disease transmission control, proposing new approaches and models of treatment based also on remote interaction techniques which will then retain their usefulness even at the end of the current emergency period.
\end{abstract}

Keywords: COVID-19; pandemics; pediatric dentistry; oral health prevention

\section{Introduction}

Prevention of oral health in children represents the gold standard towards which health professionals specialized in pediatric dentistry should always be oriented. This is even more true in times of health emergency such as the one we are going through today in which the WHO first declared the COVID-19 epidemic a Public Health Emergency of International Concern [1] and then recognized it as a pandemic [2].

It is precisely during the COVID-19 epidemic period that an adequate management of the oral health of children becomes of crucial importance by implementing specific protocols relating both to the pathologies of the oral cavity that normally do not represent an emergency and to those clinical situations that fall within the category of pediatric dental emergencies.

In both conditions, the main objective is to limit the spread of the epidemic and the onset of cross-infections. Therefore, not only are rigorous and highly effective infection control protocols urgently needed in the dental environments of the regions affected by COVID-19, but it is also essential to work on remote communication and education aimed at maintaining the oral health of children. 


\section{COVID-19 Risk Factors Associated with Pediatric Dental Treatment}

Genetic and epidemiological research reports that the COVID-19 epidemic started with a single transmission from animal to man, followed by an important human-to-human transmission [3,4]. The human-to-human transmission of COVID-19 occurs mainly through respiratory droplets in air suspension and aerosol and through direct or indirect contact $[5,6]$. Mother-infant vertical transmission has not yet been confirmed $[7,8]$.

In dental settings, oral fluids from the patient or contaminated dental instruments or environmental surfaces create a potential way of spreading the virus to the operators and to other patients. Standard dental procedures that include the use of rotating instruments such as the high-speed turbine handpiece and the use of scalers for oral hygiene are associated with the generation of large quantities of aerosols and droplets from the saliva and blood of the patient. These can remain suspended in the air for long periods (hours) before settling on the environmental surfaces and on the medical instruments or before penetrating the respiratory tract through the nose and mouth. Hence, it is clear how the aerosols generated during dental treatment can expose the pediatric dentist, the assistant to the chair and other patients to risk of cross infection.

A recent article [9] reports a review of the main risk factors for COVID-19 associated to pediatric dental treatments. A first way of contagion is represented by direct transmission through the respiratory droplets: the virus can be transmitted between the dentist, the dental assistant and the small patients through the droplets generated by coughing and sneezing or by the dental procedures themselves. An indirect transmission can occur when the droplets containing the virus settle on the surfaces of the instruments with consequent transmission when the pediatric dentist, the assistant in the chair and other patients come into contact with the contaminated surfaces. Similarly, the use of high-speed turbine, ultrasonic scaler and spray gun produce an aerosol of saliva, blood and other fluids that can remain in air suspension for long periods, with an increased risk of transmission.

While the previous transmission pathways are common to the treatment of any dental patient, pediatric patients present additional risks of transmission: the use of removable orthodontic appliances or auxiliary elements in fixed orthodontic therapies, such as the use of intermaxillary elastic bands, entails risks of contamination if handling is not carried out with due precautions. Another problem is related to the difficulty for the child to use/endure personal protective equipment (PPE) during medical visits. Finally, the very presence of caregivers, with whom the pediatric dentist must unavoidably interface, will increase the risk of infection.

\section{Oral Health Prevention during the COVID-19 Pandemics}

\subsection{General oral health prevention measures}

In general, the prevention of oral health in children is based on the periodicity of the check-ups and on oral hygiene education through adequate information of the parent on orodental diseases. In an epidemic context such as that from COVID-19, the difficulty of managing regular follow-ups of dental check-ups makes it essential to focus prevention on oral health education interventions that go through adequate remote information. For this purpose, it is possible to use "social" digital platforms on which the pediatric dentist can publish and disseminate behavioral guides for the protection of the oral health of children. The main goal of these guides would be to avoid, or at least minimize, the onset of unfavorable situations for the stomatognathic system, thus improving the oral health of the child.

\subsection{Prevention of carious pathology}

Prevention against the carious pathology must be based on adequate and effective home oral hygiene measures, on the use of dental floss in the tooth exchange phase associated with the completion of permanent dentition, and on correct alimentary behaviors which limit the intake of fermentable carbohydrates. It is important in this sense to inform parents about the carioprotective 
and cariogenic properties of specific foods [10]. A diet rich in fruit and vegetables not only protects from the onset of caries, thanks to a mechanical protection associated with the stimulation of saliva, but also helps to protect against the onset of gum diseases. Furthermore, during this epidemic period in which children are forced to spend most of their time at home, it is of fundamental importance to limit the consumption of soft and energy drinks since, in addition to containing high quantities of sugars, their acidic content produces dental erosion and makes the enamel tissue less resistant to the attack of cariogenic bacteria.

Early Childhood Caries (ECC) affects the age group between 3 and 5 years and has a rapidly worsening clinical evolution with easy onset of local abscess infectious complications associated with painful symptoms. The treatment of these complications requires emergency interventions and would therefore expose the child to potential risks of contagion from COVID-19. ECC prevention is therefore of fundamental importance and requires the interruption of incorrect alimentary habits such as the administration, especially during the night, of bottles filled with fermentable liquids containing carbohydrates, rather than pacifiers dipped in honey or sugar, erroneous expedients often adopted by parents to encourage the sleep of the child.

Finally, it should not be forgotten that preservation of the oral health of the parents also has a protective effect for the child as the risk of bacterial mother-child transmission is high in the age group between 3 and 5 years.

\subsection{Other aspects of pediatric dental prevention}

The need to spend the whole day in a home setting can stimulate more lively play modes in the children, with an increased risk of traumatic events affecting the dental elements and the consequent need for urgent interventions that increase the risk of contagion from COVID-19. Parents should therefore be instructed on the need for careful supervision of the child's activities, possibly recommending the use of protective mouthguards of the type usually indicated for contact sports.

In general, correct oral health must always be combined with a healthy lifestyle, thus contributing to a good general health. Therefore, physical activity at home, sleeping for an adequate number of hours, and proper nutrition and hydration throughout the day are strongly recommended.

\section{Dental Management during the COVID-19 Outbreak}

\subsection{Management of orodental pathologies that do not represent an emergency}

During the COVID-19 epidemic and together with all other medical activities, routine dental work in Italy was suspended and postponed in relation to the progress of the epidemic situation, with the recommendation of limiting dental interventions to emergencies only [11]. Hence, the need to be able to follow the oral health status of children only through adequate remote interaction with parents, who must be properly trained on the recognition of diseases that do not require immediate intervention and which can therefore be managed with palliative measures, waiting to be treated in an outpatient setting at the end of the epidemic emergency.

In agreement with previous literature [9], we list here several clinical situations that do not require emergency treatment and can therefore be remotely managed.

1. Deciduous or permanent teeth affected by previous carious lesions and treated with temporary dressings: in this case if the dressings were to decement from the prepared cavity, it is recommended to keep the cavity always free of food debris through careful removal with mechanical brushing after meals to prevent the onset of painful symptoms. It is also recommended to avoid too hot or too cold foods that could trigger the onset of painful symptoms if the original treatment involved the removal of carious dentin-enamel tissue.

2. If the dressing concerns an endodontically treated deciduous or permanent tooth and the spontaneous removal of the dressing paste occurs, home treatment may involve washing the 
endodontic cavity with water diluted with hydrogen peroxide by means of a special syringe without a needle, followed by application of a cotton pellet during the child's meals.

3. Chronic periapical periodontitis can occur with dental pain when chewing. For temporary control of symptoms, antibiotic therapy with amoxicillin or cephalosporin and pain relievers such as ibuprofen are recommended, which are adjuvants to temporarily relieve symptoms and postpone dental treatment.

4. Delays of deciduous teeth exfoliation with their persistence in the arch, in conjunction with the simultaneous eruption of the corresponding permanent tooth are quite common occurrences. In this case, parents should be advised to encourage the child to chew hard consistency foods such as raw fruit and vegetables, that can stimulate the loss of the deciduous tooth by mechanically inducing its complete removal from the alveolar support.

5. Eruptive gingivitis of the permanent first molar is an additional clinical condition, which can be managed during this period. It manifests itself with swelling, edema and redness of the gum in distal position to the erupting first permanent molar. This condition affects school-aged children, on average between 6 and 7 years. The advice to give to the parents is to use cleaning swabs that help the removal of food debris in the gingival bag between tooth and gum, by rinsing with anti-inflammatory mouthwashes alternating during the day with local chlorhexidine antiseptic sprays, in order to reduce the inflammatory state and the painful symptoms.

6. Malocclusions associated with crowding of the dental elements and with overjet and overbite alterations do not represent an emergency. Parents must be instructed to postpone the correction to the end of the epidemic. In case of increased overjet, a condition most frequently associated with the risk of fractures affecting the upper incisors, it is possible to recommend the use of standard mouthguards, easily available in the pharmacy.

\subsection{Management of children undergoing orthodontic treatment}

If the child is using a removable orthodontic appliance, the first indication to the parents is linked to the correct hand hygiene measures before inserting the device into the oral cavity. According to recent studies [12], the oral cavity has in fact a high expression of the ACE2 receptor, considered the main host cell receptor for the SARS-CoV-2 virus. This fact also underlines the importance of a correct hygiene associated with the management of orthodontic devices, which must be carefully sanitized before each use and stored in the appropriate box after use.

If Schwarz removable plates are used, a broken retention hook, such as the Adams hook that normally fits on the first permanent molar, is a not unusual occurrence. In this case, if the entire hook breaks from the resin section of the appliance, the child can continue to wear the device as long as the retention is preserved. Alternatively or in case of doubt, it is advisable to limit the use of the device during the daytime hours only, always under parental supervision, postponing the repair of the device at the end of the epidemic.

For fixed orthodontic devices cemented on the palatal arch, such as the rapid palatal expander, it is recommended to temporarily suspend the activations in order to avoid carrying out incongruous maneuvers that can facilitate the detachment of the device from the dental surfaces. Given the current epidemic, it should be forcefully reiterated to the parents that the child must avoid eating viscous foods, such as caramel or chewing gum, or hard foods that can favor the partial detachment of the device, thus triggering an emergency situation that requires an immediate intervention by the pediatric orthodontist.

In the case of fixed multi-bracket therapy, the arch may slide and move distally to the cemented tube on the molars. In this case, especially when light NiTi wires are in use, if the child reports a feeling of discomfort and puncture on the gum, it is possible to advise the parent to manually reposition the arch by sliding it towards the teeth most mesial to the molar using the fingertips of thumb and index. If a bracket decements from the dental surface and rotates by $180^{\circ}$ 
with respect to the tooth while remaining tied to the arch, the parent can reposition it manually, postponing the re-cementation to the end of the epidemic.

\subsection{Management of orodental pathologies that represent an emergency}

Many pediatric dental emergencies require immediate treatment even during the COVID-19 outbreak. Among these, we can list, e.g., acute pulpitis, acute apical periodontitis, dental trauma and maxillofacial trauma [13,14].

The management of all pediatric dental emergencies during the epidemic must take place by adopting protective measures for healthcare personnel and for the young patient in accordance with the recommendations and guidelines related to the use of effective protocols for the prevention and control of infections referred to by the scientific literature [15-19].

\subsubsection{Personal protective measures for the pediatric dentist}

Since the main route of transmission of COVID-19 is through airborne droplets, during the epidemic period, additional protective measures with personal protective equipment (PPE) are recommended for the pediatric dentist and other healthcare professionals. These include: surgical mask, face shields, protective goggles, gloves, medical cap, and protective suits.

We can distinguish three levels of protection for the pediatric dentist:

- $\quad$ standard primary protection for staff in a clinical context: wear a disposable work cap; disposable surgical mask; work clothes with white coat; protective goggles or face shields and disposable latex or nitrile gloves;

- secondary or advanced protection: disposable cap, disposable surgical mask, protective goggles, face shield, white work coat with disposable or external surgical insulation clothing and disposable latex gloves;

- tertiary or enhanced protection when coming into contact with suspected or confirmed patients with COVID-19 infection: although a patient with suspected or confirmed infection with COVID-19 should not be treated, in the unlikely event that this happens and the pediatric dentist cannot avoid close contact, special protective clothing is required. If protective clothing is not available, a lab coat with an external disposable protective suit should be worn. In addition, disposable caps, protective goggles, face shields, disposable surgical masks, disposable latex gloves and waterproof shoe covers should be worn.

\subsubsection{Patient evaluation}

In this phase it is necessary to make a recording of the temperature of both the child and their carers and to carry out an anamnestic evaluation to investigate not only trips to geographic areas affected by the epidemic of COVID-19 made in the 14 days preceding the dental visit but also if there is a history of contact with COVID-19 patients or suspected such [6]. The child should be accompanied by a minimum number of people. In addition to measuring the temperature, medical protective masks should be provided to patients and their carers.

\subsubsection{Hand hygiene}

The reinforcement of good hand hygiene of the reception staff, of the patient, and of the medical staff is of fundamental importance especially in the period of transmission of the epidemic from COVID-19. Given the transmission characteristics of the SARS-CoV-2 virus, a bad compliance of hand washing in the dental practice poses a challenge to controlling infection during the epidemic period. A Chinese study [20] recommends the "two before and three after" technique as a standard hand hygiene procedure, emphasizing that oral professionals should wash their hands before examining the patient, before dental procedures, after direct contact with the patient, after touching the environment without previous disinfection, and after touching the patient's oral mucosa and skin or coming into contact with saliva and oral fluids.

\subsubsection{Mouthwash rinses}


Recent studies [21] indicate that chlorhexidine, the most commonly used mouthwash in dental studies, is not effective against the SARS-CoV-2 virus. On the other hand, the virus appears to be vulnerable to oxidation: it is therefore possible to let the child rinse with dedicated antiseptic solutions to reduce the oral bacterial load.

\subsubsection{Recommendations for treatment}

- Get parents to leave the operating room.

- Use four-handed dentistry techniques.

- Use a high volume aspirator to minimize droplets and aerosol during high-speed turbine operation.

- Isolate the operating field with the rubber dam in order to minimize the production of aerosols contaminated with blood and saliva, especially during the treatment of the pulpits.

- Use high-speed turbines with anti-retraction valve, which significantly reduces the return flow of oral bacteria.

\subsubsection{Management of traumatic orodental injuries}

Traumatic pathology has a prevalence that varies between $6.1 \%$ and $62.1 \%$ in individuals of preschool age [22] and between $5.3 \%$ and $21 \%$ of school age [23]. It is therefore likely that it can present itself as an emergency to be managed during the COVID-19 epidemic. The condition associated with a favorable prognosis is represented by the immediate reimplantation of an avulsed permanent tooth. In this case the success rate is associated with the conservation of the tooth in physiological solution or milk or saliva taking care to rinse it first with running water to decontaminate it before its introduction into the alveolus. In case of dislocation, the emergency treatment consists in repositioning and splinting with the adjacent dental elements. In general, the planning of the treatment of dental fractures, dislocation or dental avulsion depends on the age, the traumatic severity of the dental tissue, the development of the apex and the duration of the dental avulsion [24-26]. If the patient presents with contusion of the soft tissues of the face, debridement with removal of torn and contaminated tissues, disinfection and suturing is necessary. Patients with maxillofacial lesions require instead immediate hospitalization.

\subsubsection{General recommendations}

The environment where the dental treatments are carried out must be properly disinfected and always well ventilated. After any type of treatment it is necessary to proceed to an adequate disinfection of the environment.

\section{Conclusions}

In the current context of epidemiological emergency linked to the COVID-19, it is necessary to re-evaluate the pediatric dentist's activities taking into account the challenges in terms of contagion containment. In this sense, the possibility of remote education of parents must be considered both as regards the general prevention measures for oral health, and as regards the home management of milder oral pathologies for which direct intervention of the specialist in pediatric dentistry is not necessary or can be postponed. On the other hand, if, in the presence of dental emergencies, immediate intervention is required, the observance of strict protection protocols of the subjects involved and environmental disinfection becomes crucial in order to minimize the risk of cross infection.

The end of the pandemic will have to mark the beginning of new methods of approach and management in pediatric dentistry. The smart technological systems, that during the pandemic period blossomed to become the most powerful remote communication tool, could be of great help as standard projection tool for educational material on oral health in children, especially in school age, who are treated in outpatient clinics, boosting and strengthening the approach in pediatric dentistry and the children's motivation for oral health. On the other hand, the specialists in pediatric dentistry will have matured and strengthened their dedication to the practice of this 
medical specialty in the post-pandemic period, not only by improving and modernizing the approach techniques, but also by proposing new models of treatment that may include the use of remote controls through special platforms, with practical guides dedicated to parents, in order to monitor and preserve the great heritage of general health, of which oral health is an important component.

Author Contributions: Conceptualization, Valeria Luzzi and Antonella Polimeni; Investigation, Valeria Luzzi; Methodology, Valeria Luzzi, Gaetano Ierardo, Maurizio Bossù and Antonella Polimeni; Project administration, Valeria Luzzi; Supervision, Antonella Polimeni; Validation, Gaetano Ierardo and Maurizio Bossù; Writing original draft, Valeria Luzzi; Writing - review \& editing, Valeria Luzzi, Gaetano Ierardo, Maurizio Bossù and Antonella Polimeni.

Funding: This research received no external funding.

Conflicts of Interest: The authors declare no conflict of interest.

\section{References}

1. World Health Organization. Statement on the second meeting of the International Health Regulations (2005) Emergency Committee regarding the outbreak of novel coronavirus (2019-nCoV). Available online: https://www.who.int/news-room/detail/30-01-2020-statement-on-the-second-meeting-of-the-international -health-regulations-(2005)-emergency-committee-regarding-the-outbreak-of-novel-coronavirus-(2019-nco v)

2. World Health Organization. WHO Director-General's opening remarks at the media briefing on COVID-19 $\quad$ - $11 \quad 2020 . \quad$ March 2 online: https://www.who.int/dg/speeches/detail/who-director-general-s-opening-remarks-at-the-media-briefingon-covid-19---11-march-2020

3. Chan JF, Yuan S, Kok KH, et al. 2020. A familial cluster of pneumonia associated with the 2019 novel coronavirus indicating person-to-person transmission: a study of a family cluster. Lancet. 2020, 395(10223):514-523.

4. Del Rio C, Malani PN. 2019 novel coronavirus-important information for clinicians. JAMA. 2020, [epub ahead of print 5 Feb 2020] in press. doi:10.1001/jama.2020.1490.

5. The Chinese Preventive Medicine Association. An update on the epidemiological characteristics of novel coronavirus pneumonia (COVID-19). Chin J Epidemiol. 2020, 41(2):139-144.

6. World Health Organization. Questions and answers on coronaviruses [accessed 2020 Feb 26]. Available online: https://www.who.int/news-room/q-a-detail/q-acoronaviruses

7. Chen H, Guo J, Wang C, et al. 2020. Clinical characteristics and intrauterine vertical transmission potential of COVID-19 infection in nine pregnant women: a retrospective review of medical records. Lancet 2020, [epub ahead of print 12 Feb 2020] in press. doi:10.1016/S0140-6736(20)30360-3.

8. Zhu H, Wang L, Fang C, et al. Clinical analysis of 10 neonates born to mothers with 2019-nCoV pneumonia. Transl Pediatr. 2020, 9(1):51-60. doi:10.21037/tp.2020.02.06.

9. Wang Y, Zhou CC, Shu R, Zou J. [Oral Health Management of Children during the Epidemic Period of Coronavirus Disease 2019]. Sichuan Da Xue Xue Bao Yi Xue Ban. 2020 Mar;51(2):139-145. doi: 10.12182/20200360506. Review. Chinese.

10. Trottini M, Bossù M, Corridore D, et al. Assessing risk factors for dental caries: a statistical modeling approach. Caries Res. 2015, 49(3):226-35. doi: 10.1159/000369831. Epub 2015 Mar 4.

11. Gazzetta Ufficiale della Repubblica Italiana. DECRETO-LEGGE 9 marzo 2020, n. 14. Disposizioni urgenti per il potenziamento del Servizio sanitario nazionale in relazione all'emergenza COVID-19. (20G00030). GU Serie Generale n.62 del 09-03-2020.

12. $\mathrm{Xu} \mathrm{H}$, Zhong L, Deng J, et al. High expression of ACE2 receptor of 2019-nCoV on the epithelial cells of oral mucosa. Int J Oral Sci. 2020 Feb 24;12(1):8. doi: 10.1038/s41368-020-0074-x.

13. Hammel J and Fischel J. Dental emergencies. Emerg Med Clin North Am. 2019, 37(1): 81-93.

14. Abbott P. Traumatic dental injuries are now the 5th most prevalent disease/injury in the world - But they are being neglected!! Dent Traumatol. 2018, 34(6): 383.

15. Meng L, Hua F, Bian Z. Coronavirus Disease 2019 (COVID-19): Emerging and Future Challenges for Dental and Oral Medicine. J Dent Res. 2020, Mar 12:22034520914246. doi: 10.1177/0022034520914246. [Epub ahead of print]. 
16. Liu T, Yuan WJ, Wang XD, et al. [Nursing strategy of oral and maxillofacial trauma emergency during epidemic situation of the novel coronavirus pneumonia]. China J Oral Maxillofac Surg. 2020,18(3):198-203. Chinese.

17. Kohn WG, Collins AS, Cleveland JL, et al.; Centers for Disease Control and Prevention. Guidelines for infection control in dental health-care settings. 2003. Available online: https://www.cdc.gov/mmwr/preview/mmwrhtml/rr5217a1.htm

18. Li R, Leung K, Sun F, Samaranayake L. Severe acute respiratory syndrome (SARS) and the GDP. Part II: implications for GDPs. Br Dent J. 2004, 197(3):130-134.

19. Samaranayake LP, Peiris M. Severe acute respiratory syndrome and dentistry: a retrospective view. J Am Dent Assoc. 2004, 135(9):1292-1302.

20. Peng X, Xu X, Li Y, et al. Transmission routes of 2019-nCoV and controls in dental practice. Int J Oral Sci. 2020 Mar 3;12(1):9. doi: 10.1038/s41368-020-0075-9.

21. National Health Commission of the People's Republic of China. Guideline for the Diagnosis and Treatment of Novel Coronavirus Pneumonia (5th edition). 2020. Available online: http://www.nhc.gov.cn/xcs/zhengcwj/202002/3b09b894ac9b4204a79db5b8912d4440.shtml

22. Hasan AA, Qudeimat MA, Andersson L. Prevalence of traumatic dental injuries in preschool children in Kuwait - a screening study. Dental Traumatology. 2010, 26:346-350.

23. Faus-Damia M, Alegre-Domingo T, Faus-Matoses I, et al. Traumatic dental injuries among schoolchildren in Valencia, Spain. Medicina Oral, Patologia Oral, Cirugia Bucal. 2011, 16:E292-E295.

24. Andersson L, Andreasen JO, Day P, et al. International Association of Dental Traumatology guidelines for the management of traumatic dental injuries: 2. Avulsion of permanent teeth. Dent Traumatol, 2012, 28(2): 88-96.

25. DiAngelis AJ, Andreasen JO, Ebeleseder KA, et al. International Association of Dental Traumatology guidelines for the management of traumatic dental injuries: 1. Fractures and luxations of permanent teeth. Dent Traumatol. 2012, 28(1):2-12.

26. Malmgren B, Andreasen JO, Flores MT, et al. International Association of Dental Traumatology guidelines for the management of traumatic dental injuries: 3. Injuries in the primary dentition. Dent Traumatol. 2012, 28(3):174-182. 